\title{
Vibrational Spectroscopy of Gas-Phase Metal-Carbide Clusters and Nanocrystals
}

\author{
Deniz van Heijnsbergen, ${ }^{1}$ Gert von Helden, ${ }^{1}$ Michael A. Duncan, ${ }^{2}$ André J. A. van Roij, ${ }^{1}$ and Gerard Meijer ${ }^{1}$ \\ ${ }^{1}$ Department of Molecular and Laser Physics, University of Nijmegen, \\ Toernooiveld 1, NL-6525 ED Nijmegen, The Netherlands \\ and FOM Institute for Plasma Physics Rijnhuizen, Edisonbaan 14, NL-3430 BE Nieuwegein, The Netherlands \\ ${ }^{2}$ Department of Chemistry, University of Georgia, Athens, Georgia 30602
}

(Received 2 August 1999)

Neutral $\mathrm{Ti}_{8} \mathrm{C}_{12}$ and $\mathrm{Ti}_{14} \mathrm{C}_{13}$ clusters are produced in the gas phase with pulsed-nozzle laser vaporization. Infrared multiphoton excitation with a pulsed free-electron laser results in thermionic electron emission for these clusters, and the parent molecular ions are detected. Multiphoton ionization is strongly enhanced on vibrational resonances of these clusters, making it possible to observe infrared resonance-enhanced multiphoton ionization spectra. These spectra indicate $\mathrm{C}-\mathrm{C}$ bonding for the "metcars" while $\mathrm{Ti}_{14} \mathrm{C}_{13}$ has remarkable similarities to bulk TiC.

PACS numbers: $36.40 .-\mathrm{c}, 33.20 . \mathrm{Ea}, 33.80 . \mathrm{Rv}, 61.46 .+\mathrm{w}$

Laser vaporization in pulsed-nozzle sources has made it possible to produce a fantastic variety of atomic clusters $[1,2]$. For carbon clusters, variations on these methods made it possible to produce $\mathrm{C}_{60}$ and the related fullerenes and nanotubes in macroscopic quantities and to study them using conventional spectroscopy [3]. However, most clusters produced in the gas phase, especially those containing metals, remain largely uncharacterized. Among these are the fascinating transition metal-carbide clusters, which preferentially form specific stoichiometries such as the $\mathrm{M}_{8} \mathrm{C}_{12}$ "met-cars" species [4-6] and the $\mathrm{M}_{14} \mathrm{C}_{13}$ "nanocrystals" [7,8]. We present here infrared spectra for size-selected titanium-carbide clusters in the gas phase, giving unique and direct information on their structure. Neutral gas-phase titanium-carbide clusters are excited to internal energies at which they efficiently undergo delayed ionization using a widely tunable infrared free electron laser. The excitation process is strongly enhanced on vibrational resonances, and monitoring the ion signal as a function of laser wavelength yields vibrational spectra. The spectra indicate that $\mathrm{Ti}_{8} \mathrm{C}_{12}$ has $\mathrm{C}-\mathrm{C}$ bonding while $\mathrm{Ti}_{14} \mathrm{C}_{13}$ has remarkable similarities to bulk TiC, consistent with the cage and crystallite structures previously suggested [4-8].

The $\mathrm{M}_{8} \mathrm{C}_{12}$ met-cars clusters were first reported by Guo and co-workers for the early transition metals $\mathrm{Ti}, \mathrm{V}, \mathrm{Zr}$, and $\mathrm{Hf}[4,5]$. Later studies showed that this stoichiometry is also preferred for other transition metals ( $\mathrm{Fe}, \mathrm{Cr}, \mathrm{Mo}$ ) [6]. Guo et al. first proposed a structure in which both metal and carbon are present in the wall of a symmetric cage with 12 five-membered rings on its surface $\left(T_{h}\right.$ symmetry) [4]. More recent theoretical work [9-12] has suggested a structure with $T_{d}$ (tetrahedral) symmetry, which has an inner set of four equivalent metal atoms, an outer set of four metal atoms capping the threefold surfaces of the inner set, and six $\mathrm{C}_{2}$ moieties spanning diagonally across the surface metal atoms. Unlike the $T_{h}$ structure, the $T_{d}$ configuration gains additional stability through metal-metal bonding. Both structures suggest ionic bond- ing between partially positive metals and partially negative $\mathrm{C}_{2}$ groups. Chemisorption reactivity studies have suggested either eight or four equivalent metal atoms on the surface depending on conditions $[13,14]$, while negative ion photoelectron spectra are consistent with the orbital occupation expected for the $T_{d}$ structure $[15,16]$. Ion mobility measurements determined that the structure is approximately spherical but could not distinguish between the proposed symmetries [17]. The $\mathrm{M}_{14} \mathrm{C}_{13}$ cluster was suggested to have a $3 \times 3 \times 3$ nanocrystalline cubic structure $[7,8]$, which is supported by theory $[12,18]$. Neither the $\mathrm{M}_{8} \mathrm{C}_{12}$ nor the $\mathrm{M}_{14} \mathrm{C}_{13}$ clusters have been isolated, and there is no spectroscopic data which can confirm or disprove the proposed structures.

Laser induced multiphoton absorption and subsequent thermionic electron emission have been documented previously for several gas-phase cluster systems including metals [19,20], fullerenes [21,22], and the present metal carbides [23]. Multiphoton absorption causes internal heating of the cluster, and statistical randomization of this energy takes place. When there is sufficient excess energy, dissociation may occur. However, in clusters with strong bonding and a relatively low ionization energy, electron emission can become competitive with dissociation. Thermionic emission has been observed with both visible and infrared lasers, consistent with this mechanism. The spectroscopic utility of infrared laser induced thermionic emission has recently been demonstrated for $\mathrm{C}_{60}$ [22]. In these studies, a significant enhancement in the ionization efficiency is observed when photons are absorbed into IR-allowed vibrational resonances. Although the ionization process is complex, the resulting infrared resonance-enhanced multiphoton ionization (IR-REMPI) spectrum bears close resemblance in peak position and relative intensity to the conventional infrared absorption spectrum. The success of IR-REMPI spectroscopy for the fullerenes suggests that it may be possible to employ this method for infrared spectroscopy of metal clusters produced in situ in low density molecular beams. 
Titanium-carbide clusters were chosen for this initial study because of the intriguing structures proposed for these species and because they have the required strong bonding (about 5-6 eV per atom) [9-12] and low ionization energies (4-5 eV) [24]. As mentioned above, thermionic emission has already been observed for $\mathrm{Ti}_{8} \mathrm{C}_{12}$ [23].

In the present experiments, titanium-carbide clusters are produced by vaporizing a solid rod of titanium in an expansion containing $1 \%-5 \% \mathrm{CH}_{4}$ (or ${ }^{13} \mathrm{CH}_{4}$ ) seeded in argon. The contents of the molecular beam are sampled with a reflectron time-of-flight mass spectrometer located $20 \mathrm{~cm}$ downstream from the source. The acceleration plates in the spectrometer are pulsed to extract ions from the molecular beam, and the timing between the cluster source, ionization laser, and acceleration plates are all independently adjusted with delay generators. Ions produced directly in the source plasma are blocked with an electric field $(500-1000 \mathrm{~V} / \mathrm{cm})$ perpendicular to the molecular beam located $5 \mathrm{~cm}$ prior to the ion extraction region. The source conditions are first optimized to produce the desired metal-carbide species using ultraviolet laser ionization of the neutral species in the beam with a $\mathrm{KrF}$ excimer laser at $248 \mathrm{~nm}$. Infrared excitation is performed with the "Free Electron Laser for Infrared Experiments" (FELIX) [25] in the 6.0 to $25 \mu \mathrm{m}$ wavelength region (1670 to $400 \mathrm{~cm}^{-1}$ ). The laser output consists of a "macropulse" of about $5 \mu \mathrm{sec}$ duration. The macropulse is composed of a series of picosecond duration "micropulses" spaced by one nanosecond. The macropulse energy is $20-30 \mathrm{~mJ}$, while the bandwidth is about $5-10 \mathrm{~cm}^{-1}$ in this wavelength region. The IR beam is focused into the ionization region of the mass spectrometer with a $7.5 \mathrm{~cm}$ focal length gold mirror, where it intersects the molecular beam. The molecular beam is formed when a pulse of argon expansion gas is synchronized to the metal vaporization laser pulse, and the pulsed infrared beam is delayed to intersect this gas burst in the mass spectrometer. The acceleration voltages in the mass spectrometer are pulsed with a variable delay $(1-3 \mu \mathrm{s})$ after the end of the laser pulse.

The laser vaporization process produces a molecular beam containing both neutral and ionized clusters of various metal carbides. Photoionization of the neutrals, or pulsed sampling of the cations, produces mass spectra like those reported previously, with very prominent $\mathrm{Ti}_{8} \mathrm{C}_{12}$ and $\mathrm{Ti}_{14} \mathrm{C}_{13}$ masses. However, when the ion blocking field is applied between the source and the mass spectrometer, ions produced directly in the source plasma are deflected from the molecular beam and these are not detected.

To exploit the thermionic emission process for spectroscopy, we employ infrared excitation of the molecular beam using FELIX. No ions are observed until the infrared laser is turned on at the precise time when the pulse of neutral clusters passes through the mass spectrometer. Figure 1 shows that surprisingly large ionization signals are observed following infrared excitation. In spite of the extreme laser conditions used, only a few specific ions are formed, and laser power dependence studies reveal little evidence for fragmentation. Most importantly, different clusters are produced more effectively at different wavelengths. The upper trace shows the mass spectrum when the excitation laser is tuned to $\lambda=7.3 \mu \mathrm{m}$. The most prominent feature is centered around $528 \mathrm{amu}$, which is the mass assigned previously to the $\mathrm{Ti}_{8} \mathrm{C}_{12}$ met-car cluster. ${ }^{13} \mathrm{C}$ isotope studies (needed because of the coincidence between $\mathrm{Ti}$ and $4 \mathrm{C}$ masses) confirm that this is the stoichiometry observed here. Other masses also detected in this region can then be assigned to $\mathrm{Ti}_{7} \mathrm{C}_{12}$ and $\mathrm{Ti}_{8} \mathrm{C}_{11}$. The inset shows an expanded view of the $\mathrm{Ti}_{8} \mathrm{C}_{11}$ and $\mathrm{Ti}_{8} \mathrm{C}_{12}$ mass peaks revealing the pattern expected for the distribution of titanium and carbon isotopes. $\mathrm{Ti}_{7} \mathrm{C}_{12}$ is a known fragment from the dissociation of $\mathrm{Ti}_{8} \mathrm{C}_{12}$, and this peak disappears at lower laser power, indicating that it is due to fragmentation. $\mathrm{The}^{\mathrm{Ti}} \mathrm{i}_{8} \mathrm{C}_{11}$ species has not been identified previously as a fragment ion or as a particularly stable cluster, but it shows strong thermionic emission indicating its substantial stability. At $\lambda=20 \mu \mathrm{m}$, these cluster masses are much weaker, while a new intense mass peak, also with a width determined by isotopes, is detected at $828 \mathrm{amu}$. This is the mass observed previously and assigned to the $\mathrm{Ti}_{14} \mathrm{C}_{13}$ "nanocrystal" cluster. Again, ${ }^{13} \mathrm{C}$ isotopic studies confirm that this is the stoichiometry observed here. These observations unambiguously confirm the previous observations that the neutral $\mathrm{Ti}_{8} \mathrm{C}_{12}$ cluster undergoes thermionic emission when heated [23]. However, this is the first report indicating that the $\mathrm{Ti}_{14} \mathrm{C}_{13}$

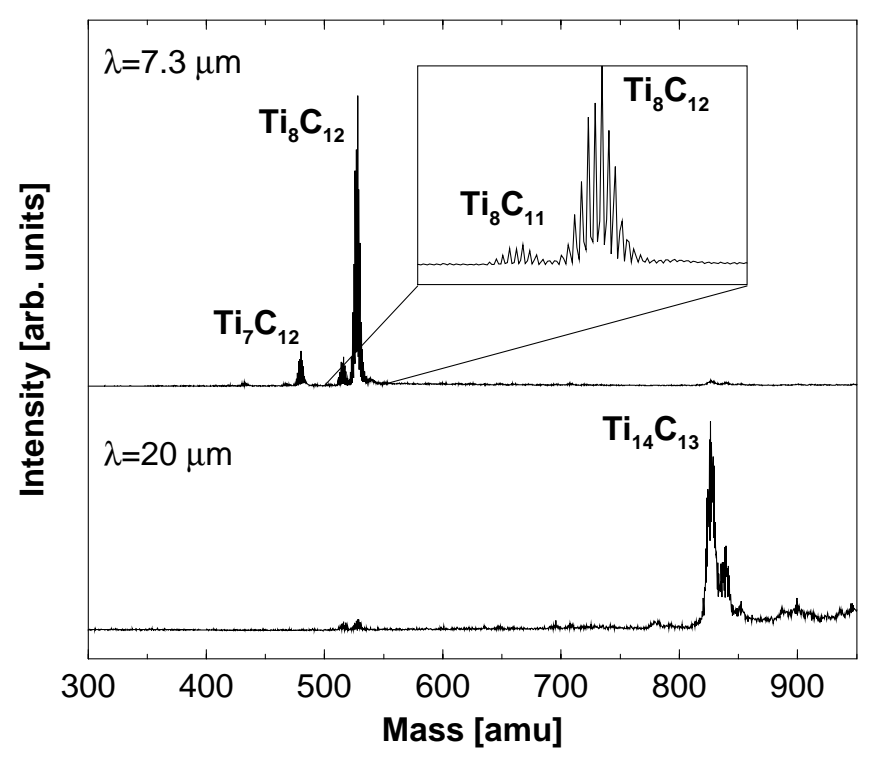

FIG. 1. Mass spectra observed after photoionization of the cluster molecular beam with the infrared free electron laser at 7.3 and $20.0 \mu \mathrm{m}$ are shown. The upper trace shows the preferential ionization of the $\mathrm{Ti}_{8} \mathrm{C}_{12}$ cluster ion and others near this mass at $7.3 \mu \mathrm{m}$. The inset shows the experimental isotope distributions, which agree very well with the ones expected for those stoichiometries. The lower frame shows the preferential formation of the $\mathrm{Ti}_{14} \mathrm{C}_{13}$ ion at the $20.0 \mu \mathrm{m}$ wavelength. 
nanocrystal cluster exhibits efficient thermionic emission as well and must therefore be exceptionally stable as a neutral.

Variation of the time delay between the infrared laser pulse and the ion extraction pulse shows that the ionization signal increases steadily throughout the FELIX macropulse and then drops off exponentially with a time constant of $2-3 \mu \mathrm{sec}$ after the laser pulse ends. For thermionic emission to be efficient, ionization in this time frame is believed to require vibrational energy well in excess of the ionization threshold. It is therefore quite remarkable that such a sequential absorption of multiple photons can occur during a single macropulse from the laser for molecules passing through the sub-mm diameter laser focus at the molecular beam velocity $(600 \mathrm{~m} / \mathrm{s})$. The density of such cluster beams is difficult to estimate due to the uncertainties in the amount of vaporized material and the efficiency of its growth into specific cluster molecules. A rough estimate suggests that the density of clusters is about $10^{8}-10^{10} \mathrm{~cm}^{-3}$ in the ionization region. The irradiated volume of the cluster beam is in the order of $10^{-4} \mathrm{~cm}^{-3}$. The efficiency of the process must therefore be relatively high (on the order of a percent) to detect the low density of sample present in the molecular beam and to produce ion signals so large that a recognizable mass spectrum is evident on the oscilloscope for each laser shot. This measurement thus demonstrates that the infrared multiphoton ionization process has enough sensitivity to be useful in low density media.

The most compelling aspect of the infrared ionization process is its wavelength dependence. Figure $2 \mathrm{a}$ shows the spectra of three selected clusters as the infrared ionization laser is tuned through the region of 400 to $1670 \mathrm{~cm}^{-1}$. It is immediately apparent that there is a strong wavelength dependence to the ionization yield and that this wavelength dependence is different for the different cluster masses measured. The $\mathrm{Ti}_{8} \mathrm{C}_{12}$ met-car cluster has a strong resonance centered at $1395 \mathrm{~cm}^{-1}$ (1345 $\mathrm{cm}^{-1}$ for the ${ }^{13} \mathrm{C}$ cluster), while the $\mathrm{Ti}_{14} \mathrm{C}_{13}$ cluster has no measurable resonance in this wavelength region.

In previous work on $\mathrm{C}_{60}$ [22], the IR-REMPI process was clearly associated with the known infrared absorption spectrum. In the present case, there are no infrared spectra of these clusters with which to compare. However, the frequencies measured can be associated with vibrational resonances for specific structural patterns in these clusters. The $1395 \mathrm{~cm}^{-1}$ band in $\mathrm{Ti}_{8} \mathrm{C}_{12}$ is logically associated with a $\mathrm{C}-\mathrm{C}$ stretching mode. $\mathrm{C}-\mathrm{C}$ bonding is expected for all of the structures proposed for the met-cars clusters, but there is no previous experimental evidence for the presence of those $\mathrm{C}_{2}$ units. By this same reasoning, the $\mathrm{Ti}_{8} \mathrm{C}_{11}$ cluster with a resonance at $1350 \mathrm{~cm}^{-1}\left(1305 \mathrm{~cm}^{-1}\right.$ for the ${ }^{13} \mathrm{C}$ cluster) can be concluded to have a similar kind of $\mathrm{C}-\mathrm{C}$ bonding. The distinctly different spectrum observed for this cluster confirms that it is not a fragment of $\mathrm{Ti}_{8} \mathrm{C}_{12}$, but that it has a related structure. The $\mathrm{Ti}_{14} \mathrm{C}_{13}$ has no de-

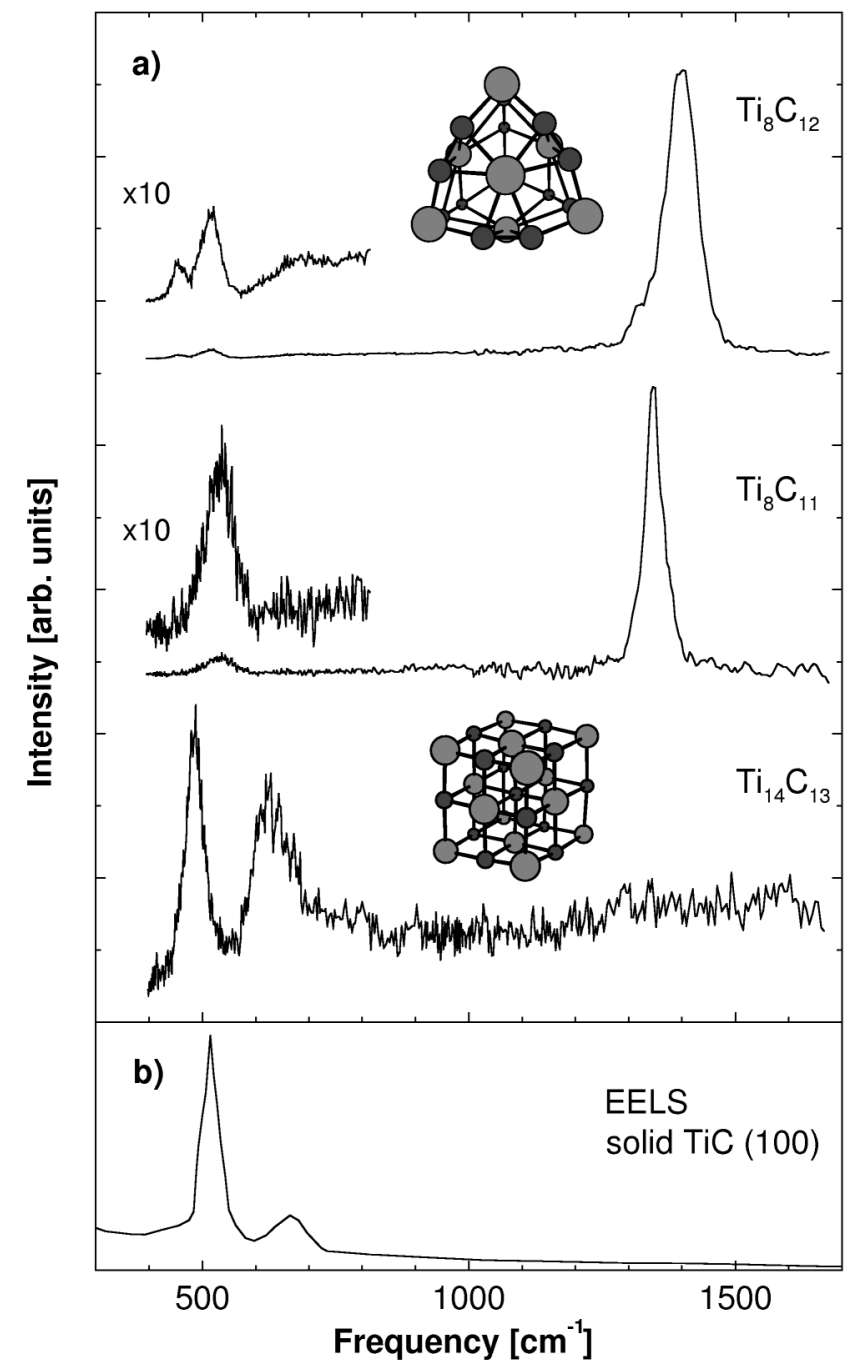

FIG. 2. (a) The infrared resonance-enhanced multiphoton ionization spectra obtained for the $\mathrm{Ti}_{8} \mathrm{C}_{12}, \mathrm{Ti}_{8} \mathrm{C}_{11}$, and $\mathrm{Ti}_{14} \mathrm{C}_{13}$ clusters are shown. In each spectrum the indicated parent molecular ion yield is measured while tuning the infrared wavelength. The structures proposed previously for $\mathrm{Ti}_{8} \mathrm{C}_{12}$ and $\mathrm{Ti}_{14} \mathrm{C}_{13}$ are shown in the figure. (b) The EELS spectrum of the rock-salt TiC (100) surface, as measured by Oshima et al. [26], is shown.

tectable resonances in this high frequency region, implying that it has no $\mathrm{C}-\mathrm{C}$ bonding. This is expected to be true for the proposed nanocrystal structure for this cluster. In the fcc lattice, there is M-C bonding, but no metalmetal or carbon-carbon bonding. All three of these clusters have resonances in the $400-600 \mathrm{~cm}^{-1}$ region. $\mathrm{Ti}_{8} \mathrm{C}_{12}$ has resonances at 520 and $455 \mathrm{~cm}^{-1}\left(500\right.$ and $450 \mathrm{~cm}^{-1}$ for the ${ }^{13} \mathrm{C}$ cluster), with a possible weak band around $660 \mathrm{~cm}^{-1}$. $\mathrm{Ti}_{8} \mathrm{C}_{11}$ has a single resonance at $535 \mathrm{~cm}^{-1}$ $\left(520 \mathrm{~cm}^{-1}\right.$ for the ${ }^{13} \mathrm{C}$ cluster). The $\mathrm{Ti}_{14} \mathrm{C}_{13}$ cluster has two resonances in the longer wavelength region at 630 and $485 \mathrm{~cm}^{-1}$ (610 and $475 \mathrm{~cm}^{-1}$ for the ${ }^{13} \mathrm{C}$ cluster). The low frequency features are particularly strong for the $\mathrm{Ti}_{14} \mathrm{C}_{13}$ cluster. Bulk TiC with the well-known rock-salt 
structure has surface phonon resonances in this region. In Fig. $2 b$ the electron energy loss spectrum [(EELS); specular direction] of the $\mathrm{TiC}$ (100) surface, as measured by Oshima et al. [26], is reproduced. Only two peaks, assigned to IR active optical surface phonon modes, are observed. The similarity between the IR-REMPI spectrum of the $\mathrm{Ti}_{14} \mathrm{C}_{13}$ cluster and the EELS spectrum of the TiC bulk surface is striking and is direct evidence for the proposed nanocrystalline structure of $\mathrm{Ti}_{14} \mathrm{C}_{13}$.

The results shown here represent the first infrared spectra of gas-phase metal clusters. Infrared spectroscopy is particularly problematic for these species due to the intrinsically weak absorption in this region and the limited availability of intense tunable light sources. The method presented here is uniquely suited to strongly bound clusters with low ionization energies, a condition which is met for many pure metal clusters and metal compound clusters. The IR-REMPI method using widely tunable free electron lasers therefore provides unprecedented opportunities to probe the structures and dynamics of sizeselected metal, nonmetal, and semiconductor atomic clusters in the isolated gas-phase environment.

We gratefully acknowledge the support by the "Stichting voor Fundamenteel Onderzoek der Materie" (FOM) in providing the required beam time on FELIX and greatly appreciate the skillful assistance of the FELIX staff. This work is part of the research program of the FOM, which is supported financially by the "Nederlandse Organisatie voor Wetenschappelijk Onderzoek" (NWO) and receives direct support from the NWO via PIONIER Grant No. 030-66-089. M. A. D. acknowledges support from the U.S. Air Force Office of Scientific Research Grant No. F49620-97-1-0042.

[1] Clusters, Series of commentaries and articles [Science 271, 920 (1996)].

[2] A. W. Castleman, Jr. and K. H. Bowen, Jr., J. Phys. Chem. 100, 12911 (1996).

[3] M.S. Dresselhaus, G. Dresselhaus, and P.C. Eklund, Science of Fullerenes and Carbon Nanotubes (Academic Press, New York, 1996).
[4] B. C. Guo, K. P. Kerns, and A. W. Castleman, Jr., Science 255, 1411 (1992).

[5] B.C. Guo, S. Wei, J. Purnell, S. Buzza, and A. W. Castleman, Jr., Science 256, 515 (1992).

[6] J. S. Pilgrim and M. A. Duncan, J. Am. Chem. Soc. 115, 6958 (1993).

[7] J. S. Pilgrim and M. A. Duncan, J. Am. Chem. Soc. 115, 9724 (1993).

[8] M. A. Duncan, J. Cluster Sci. 8, 239 (1997).

[9] I. Dance, J. Chem. Soc. Chem. Commun. 1992, 1779 (1992).

[10] I. Dance, J. Am. Chem. Soc. 118, 6309 (1996).

[11] M. M. Rohmer, M. Benard, C. Henriet, C. Bo, and J. M. Poblet, J. Chem. Soc. Chem. Commun. 1993, 1182 (1993).

[12] M. M. Rohmer, C. Bo, and J. M. Poblet, J. Am. Chem. Soc. 117, 508 (1995).

[13] B. C. Guo, K. P. Kerns, and W. A. Castleman, Jr., J. Am. Chem. Soc. 115, 7415 (1993).

[14] C. S. Yeh, S. Afzaal, S. A. Lee, Y. Byun, and B. S. Freiser, J. Am. Chem. Soc. 116, 8806 (1994).

[15] L. S. Wang, S. Li, and H. Wu, J. Phys. Chem. 100, 19211 (1996).

[16] S. Li, H. Wu, and L.S. Wang, J. Am. Chem. Soc. 119, 7417 (1997).

[17] S. Lee, N. G. Gotts, G. von Helden, and M. T. Bowers, Science 267, 999 (1995).

[18] I. Dance, J. Am. Chem. Soc. 118, 2699 (1996).

[19] A. Amrein, R. Simpson, and P. Hackett, J. Chem. Phys. 95, 1781 (1991).

[20] T. Leisner, K. Athanassenas, D. Kreisle, E. Recknagel, and O. Echt, J. Chem. Phys. 99, 9670 (1993).

[21] E. E. B. Campbell, G. Ulmer, and I. V. Hertel, Phys. Rev. Lett. 67, 1986 (1991).

[22] G. von Helden, I. Holleman, G. M. H. Knippels, A. F. G. van der Meer, and G. Meijer, Phys. Rev. Lett. 79, 5234 (1997).

[23] B. D. May, S. F. Cartier, and A. W. Castleman, Jr., Chem. Phys. Lett. 242, 265 (1995).

[24] H. Sakurai and A. W. Castleman, Jr., J. Phys. Chem. 102, 10486 (1998).

[25] G. M.H. Knippels, R.F.X. A.M. Mols, A.F. G. van der Meer, D. Oepts, and P.W. van Amersfoort, Phys. Rev. Lett. 75, 1755 (1995).

[26] C. Oshima, T. Aizawa, M. Wuttig, R. Souda, S. Otani, and Y. Ishizawa, Phys. Rev. B 36, 7510 (1987). 